\section{PERCUTANEOUS CORONARY INTERVENTION VS CORONARY ARTERY BYPASS GRAFTING IN LEFT MAIN CORONARY ARTERY DISEASE: A SYSTEMATIC REVIEW AND UPDATED META-ANALYSIS}

${ }^{1} \mathrm{C}$ Hennessy, ${ }^{2} \mathrm{JA}$ Henry, ${ }^{1} \mathrm{DT}$ Brameier, ${ }^{3,4}$ RK Kharbanda, ${ }^{4,5} \mathrm{SG}$ Myerson. ${ }^{1}$ Medical School Offices, Medical Sciences Division, University of Oxford; ${ }^{2}$ Oxford University Clinical Academic Graduate School, Oxford University Hospitals Foundation Trust, OX3 9DU; ${ }^{3}$ Oxford Heart Centre, NIHR Biomedical Research Centre, Oxford University Hospitals, Oxford, UK: ${ }^{4}$ Division of Cardiovascular Medicine, Radcliffe Department of Medicine, University of Oxford, Oxford, UK; ${ }^{5}$ Department of Cardiology, Oxford University Hospitals NHS Foundation Trust, Oxford, $U K$

10.1136/heartjnl-2021-ICS.63

Aim There is still no consensus whether percutaneous coronary intervention (PCI) is as safe and effective as coronary artery bypass grafting (CABG) for left main coronary artery (LMCA) disease. Until recently, European and US guidelines recommended CABG as the standard of care for LMCA disease management. However numerous recent studies suggest that PCI with drug eluting stents (DES) has a low risk of mortality at medium and long-term follow-up after left main stenting. This has resulted in European guidelines suggesting that PCI may be effective in treating anatomically simple LMCA disease, while CABG is still the gold standard for more complex lesions. These changes have been largely informed by six RCTs which have compared PCI vs CABG in LMCA disease. However, the results of these studies have not been straightforward, with often conflicting results. The continuing lack of consensus coupled with the recent publication of 5- and 10-year data from some of these studies necessitated an updated systematic review and meta-analysis.

Methods A systematic search was performed of the MEDLINE (via PubMed), EMBASE, Cochrane and clinicaltrials.gov databases from inception to December 20th, 2020. Only RCTs which compared PCI and CABG in LMCA disease were included. Studies were required to have a minimum follow-up of one year and to report all-cause mortality as an outcome at a minimum. Selected studies were analysed using the random effects model. Outcomes measured included all-cause mortality, MACCE, myocardial infarction (MI), stroke and revascularization. Follow up points were 30 days, 1 year, 5 years and 10 years. Where MI was concerned, meta-analysis was performed twice using the originally published EXCEL MI data as well as a second time using the recently released MI data classified according to the third universal definition of MI.

Results Six RCTs with a total population of 4700 were included in the analysis. For all-cause mortality PCI was noninferior to CABG at all time points (30 days (OR 0.6), 1 year (OR 0.77), 5 years (OR 1.41), 10 years (OR 1.08). MACCE favoured PCI at 30 days (OR $0.83 \mathrm{P}<0.01)$, and CABG at 5 years $(\mathrm{OR} 1.45, \mathrm{P}<0.001)$. No difference was seen at 1 year (OR 1.20) and 10 years (1.01). There was no significant difference in $\mathrm{MI}$ occurrence between the PCI and CABG groups at 30 days (OR 0.77), 1 year (1.10), or 10 years (OR 1.01). Using the original 5-year EXCEL trial MI definition, 5year MI rates were higher for PCI than CABG (OR 1.66, P $<0.05$ ). When the re-analysed EXCEL data (using the third universal definition of $\mathrm{MI}$ ) was included instead, the 5-year data further favoured $\mathrm{CABG}$, with $7.5 \% \mathrm{MI}$ rates in the PCI group compared to $3.6 \%$ in the CABG group (OR 2.19, $\mathrm{P}<$ $0.001)$. For stroke, PCI was favourable to CABG at 30 days (OR 0.40, $\mathrm{P}<0.05$ ) and 1 year (OR $0.63 \mathrm{P}<0.001$ ), with no difference at 5 years (OR 0.86) and 10 years (OR 0.78). Revascularisation favoured CABG at 1 year (OR 2.03, $\mathrm{P}<$ 0.001 ), and 5 years (OR 1.81, $\mathrm{P}<0.001$ ), with no differences at the other time-points.

Conclusions The results of this meta-analysis show that PCI may be considered as an alternative to CABG for uncomplicated LMCA disease. However, more complex lesions may still benefit from CABG. CABG may be of benefit where future revascularisation would rather be avoided, as lower rates of revascularisation were observed in the CABG groups at 1 year and 5 years. Further clinical trials examining this research question are needed.

\section{OUTCOMES FOLLOWING SURGICAL REPAIR OF ANOMALOUS AORTIC ORIGIN OF CORONARY ARTERIES}

J Nicoletti, G Hooks, A Graham. Belfast Health and Social Care Trust, UK

\subsection{6/heartjnl-2021-ICS.64}

Introduction Although coronary arteries that arise from the opposite sinus of Valsalva have a known association with sudden cardiac death, they most commonly present to cardiologists and cardiac surgeons with symptoms of either typical or atypical angina. This retrospective study aimed to examine the outcomes of a consecutive set of patients who underwent surgical repair of their anomalous coronary arteries under the care of one cardiac surgeon from 2010 to date.

Methods The hospital cardiac surgery records were searched for keywords to identify all retrospective cases of coronary artery anomalies since 2010. Eligible patients were contacted and surveyed about their symptoms and their electronic healthcare records were checked. Only patients who

Abstract 64 Table 1 Postoperative outcomes following repair of anomalous coronary arteries in Belfast

\begin{tabular}{|c|c|c|}
\hline Postoperative Outcomes & $\begin{array}{l}\text { Number of } \\
\text { cases }\end{array}$ & $\%$ \\
\hline \multicolumn{3}{|l|}{ Symptoms } \\
\hline Resolution & 2 & $17 \%$ \\
\hline Significant improvement & 9 & $75 \%$ \\
\hline No change & 1 & $8 \%$ \\
\hline Worse & 0 & $0 \%$ \\
\hline Complications & 7 & $58 \%$ \\
\hline Return to theatre & 2 & $17 \%$ \\
\hline Multi-organ failure & 1 & $8 \%$ \\
\hline IABP insertion & 1 & $8 \%$ \\
\hline Non-union of sternum & 1 & $8 \%$ \\
\hline Surgical site infection & 1 & $8 \%$ \\
\hline Respiratory tract infection & 1 & $8 \%$ \\
\hline Atrial fibrillation & 1 & $8 \%$ \\
\hline Deep vein thrombosis & 1 & $8 \%$ \\
\hline \multicolumn{3}{|l|}{ Long term outcomes } \\
\hline Failure of graft/ & 1 & $8 \%$ \\
\hline \multicolumn{3}{|l|}{ reimplantation } \\
\hline MACE at 5 years* & 0 & $0 \%$ \\
\hline Death within 5 years* & 0 & $0 \%$ \\
\hline
\end{tabular}

